\title{
Desempenho produtivo de macieiras 'Royal Gala' e 'Fuji' submetidas a diferentes densidades de plantio e porta-enxertos
}

\author{
Adílson José Pereira( ${ }^{(1)}$ e Mateus da Silveira Pasa(1) \\ (1)Empresa de Pesquisa Agropecuária e Extensão Rural de Santa Catarina, Estação Experimental de São Joaquim, CEP 88600-000 \\ São Joaquim, SC, Brasil. E-mail: pereiraajp@hotmail.com, mateuspasa@epagri.sc.gov.br
}

Resumo - O objetivo deste trabalho foi avaliar a influência da densidade de plantio e de porta-enxertos sobre o desempenho produtivo de macieiras 'Royal Gala' e 'Fuji'. O trabalho foi realizado de 1996 a 2007, com as cultivares Royal Gala e Fuji, na região de São Joaquim, SC. Os tratamentos consistiram de dois porta-enxertos (M9 e M26) e três densidades de plantio (1.667, 2.500 e 5.000 plantas por hectare), dispostos em arranjo fatorial $2 \times 3$, com seis tratamentos por cultivar. Utilizou-se o delineamento de blocos ao acaso, com quatro repetições. $\mathrm{O}$ aumento da densidade de plantio aumenta a produtividade e diminui a produção e o número de frutos por planta, independentemente da cultivar. Além disso, o aumento da densidade de plantio reduz o vigor das plantas (seção transversal do tronco) e a massa de fruto. A produtividade induzida pelo M26 é maior do que a induzida pelo M9, porém, este produz frutos maiores em razão, possivelmente, da menor carga de frutos das plantas com esse porta-enxerto.

Termos para indexação: Malus domestica, alta densidade, controle de vigor, pomares modernos, porta-enxertos ananicantes.

\section{Yield performance of 'Royal Gala' and 'Fuji' apple trees subjected to different planting densities and rootstocks}

\begin{abstract}
The objective of this work was to evaluate the effect of planting density and rootstocks on the yield performance of 'Royal Gala' and 'Fuji' apple trees. The experiment was carried out from 1996 to 2007 with the apple cultivars Royal Gala and Fuji, in the São Joaquim region, in the state of Santa Catarina, Brazil. Treatments consisted of two rootstocks (M9 and M26), and three planting densities (1,667, 2,500, and 5,000 plants per hectare), in a $2 \times 3$ factorial arrangement, with six treatments for each cultivar. A randomized complete block design was used, with four replicates. Increasing planting densities increases yield and decreases production and number of fruit per tree, regardless of the cultivar. Moreover, higher planting densities reduce tree vigor (expressed by trunk transverse section) and fruit weight. Induced yield by M26 is greather than that by M9, but the latter produces larger fruit, possibly due to the lower crop load of the trees on this rootstock.
\end{abstract}

Index terms: Malus domestica, high density, vigor control, modern orchards, dwarfing rootstocks.

\section{Introdução}

A maçã (Malus domestica Borkh.) é a segunda fruta de clima temperado mais produzida no Brasil, e Rio Grande do Sul e Santa Catarina são seus principais estados produtores (Instituto Brasileiro de Geografia e Estatística, 2013). São vários os fatores responsáveis pelo grande desenvolvimento da cultura da macieira no país, como definição de cultivares (Gala e Fuji) e clones capazes de atender às exigências dos consumidores e 0 desenvolvimento de tecnologias de manejo adequadas (Fachinello et al., 2011). No entanto, embora existam pomares altamente tecnicizados, a produtividade média dos pomares ainda é baixa $\left(\sim 32 \mathrm{Mg} \mathrm{ha}^{-1}\right)$, em parte em razão das baixas densidades de plantio adotadas e da utilização de porta-enxertos muito vigorosos.

Nesse sentido, sistemas mais eficientes e rentáveis, como o de plantio em alta densidade, devem ser utilizados para manter a competitividade da cultura da macieira no País. As principais vantagens do adensamento de pomares são a precocidade de entrada em produção, a elevada produtividade, a alta qualidade dos frutos e o menor custo com mão de obra (Hampson et al., 2002). $\mathrm{O}$ aumento da densidade de plantio é um dos fatores mais importantes no aumento da produtividade de pomares de macieira (Petri et al., 2011); no entanto, esse aumento está associado a acréscimo considerável 
de seu custo de implantação e manejo (Sansavini et al., 2008). Kreuz et al. (2006) relatam que a rentabilidade da cultura é maior em densidades de até 3.860 plantas ha- $^{-1}$.

A densidade de cultivo da macieira geralmente é aumentada pela redução do espaçamento entre plantas, já que as operações mecanizadas de manejo impõem limites para a redução da distância nas entrelinhas (Policarpo et al., 2006). Atualmente, a densidade utilizada em plantios modernos varia de 1.000 a 6.000 plantas $\mathrm{ha}^{-1}$, com uso de até 10.000 plantas ha $\mathrm{h}^{-1}$ em alguns sistemas (Robinson, 2011a). No entanto, estudos recentes mostraram que o melhor retorno econômico é obtido com densidades de plantio entre 2.000 e 3.200 plantas ha ${ }^{-1}$ (James \& Middleton, 2011; Robinson et al., 2013).

O sucesso de plantios adensados, no entanto, depende da utilização de técnicas de manejo para controlar o tamanho das plantas (Pasa \& Einhorn, 2014), como a correta escolha do porta-enxerto. Para tal, as limitações e vantagens de cada porta-enxerto devem ser avaliadas de maneira a escolher aquele com as melhores características para cada condição específica de cultivo (Gjamovski \& Kiprijanovski, 2011). A utilização de porta-enxertos que controlem o vigor da cultivar copa é fundamental para a produção de frutas de maior qualidade e para elevada produtividade, em pomares adensados (Tworkoski \& Miller, 2007). Mundialmente, grande parte dos pomares de macieira que obtiveram sucesso nesse sistema foram plantados com porta-enxertos ananicantes, como M9 e M26 (Robinson, 2011a).

Embora alguns pomares no Brasil já utilizem maiores densidades de plantio e porta-enxertos menos vigorosos, ainda há muitos deles que adotam baixas densidades de plantio, com porta-enxertos vigorosos, como o 'Marubakaido', ou a combinação deste com filtro de M9. Embora o plantio em alta densidade com esses porta-enxertos seja possível, a sua baixa precocidade e seu vigor excessivo com o envelhecimento das plantas são grandes limitantes (Robinson, 2011a). A resistência de alguns produtores em adotar plantios adensados é decorrente, em parte, da escassez de informações quanto ao comportamento das principais cultivares sob alta densidade de plantio, nas condições específicas de cada região de cultivo.

O objetivo deste trabalho foi avaliar a influência da densidade de plantio e de porta-enxertos sobre o desempenho produtivo de macieiras 'Royal Gala' e 'Fuji'.

\section{Material e Métodos}

O trabalho foi realizado na Estação Experimental Epagri, em São Joaquim, SC (28¹7'39"S, 4955'56"W, à altitude de $1.415 \mathrm{~m}$ ), entre 1996 e 2007 . Os dados meteorológicos coletados durante a condução do experimento estão apresentados na Tabela 1 . $\mathrm{O}$ acúmulo médio de temperaturas iguais ou inferiores a $7,2^{\circ} \mathrm{C}$, na região, é de 900 horas. O solo do campo experimental é classificado como Cambissolo Húmico, de acordo com o Sistema Brasileiro de Classificação de Solos (Santos et al., 2013).

O pomar foi instalado no inverno de 1996, em área previamente corrigida e adubada, conforme a análise química do solo. Foram utilizadas mudas das cultivares Royal Gala e Fuji com haste única, ou seja, sem ramificações laterais. A estrutura de sustentação foi composta de arame, com três fios, em cada linha de plantio. As plantas foram conduzidas no sistema líder central e mantidas à altura de aproximadamente $3,5 \mathrm{~m}$. As cultivares foram plantadas à proporção de $50 \%$, ou seja, para cada fila de 'Royal Gala', plantou-se uma de 'Fuji'. O manejo do pomar foi realizado de acordo com as recomendações do sistema de produção de macieira (A cultura da macieira, 2006).

Os tratamentos consistiram de dois porta-enxertos e três densidades de plantio, arranjados em um fatorial $2 \times 3$, com o total de seis tratamentos para cada cultivar. Os porta-enxertos e as densidades de plantio foram casualizados separadamente por cultivar, uma vez que cada linha de plantio foi preenchida com apenas uma cultivar, para facilitar o manejo da área e a polinização. Portanto, o estudo constituiu-se de dois experimentos, um para cada cultivar. Os porta-enxertos avaliados eram da série inglesa "Malling" (M) - M9 e M26 -, e foram escolhidos pelo seu potencial de reduzir o vigor da cultivar de copa. As densidades de plantio foram $1.667,2.500$ e 5.000 plantas por hectare, obtidas com o uso dos espaçamentos 1,5, 1,0 e 0,5 m entre plantas, respectivamente; sempre com $4 \mathrm{~m}$ entre as linhas de plantio, independentemente dos tratamentos. Utilizouse o delineamento experimental utilizado de blocos ao acaso, com quatro repetições (parcelas). Cada parcela constituiu-se de cinco plantas, em que apenas as três plantas centrais foram utilizadas nas avaliações, tendo- 
se deixado uma planta, em cada extremidade, como bordadura.

A colheita foi realizada quando os frutos estavam no ponto de maturação comercial, com as seguintes características: índice iodo-amido de $4 / 5$; firmeza de 80-90 N; e ${ }^{\circ}$ Brix de 11-12 (A cultura da macieira, 2006). No período da colheita, todos os frutos das plantas avaliadas foram colhidos, contados e pesados. A partir desses dados, calcularam-se as seguintes variáveis: produção por planta $(\mathrm{kg})$; massa média de frutos $(\mathrm{g})$, obtida pela relação entre a produção por planta e o número de frutos; produtividade $\left(\mathrm{Mg} \mathrm{ha}^{-1}\right)$, obtida da multiplicação da produção por planta pelo número de plantas por hectare, em cada densidade de plantio. Ao final de cada ciclo vegetativo, com exceção de 1998, 2000, 2002 e 2003, para ambas as cultivares, e de 2006, para 'Fuji', a circunferência do tronco foi medida $(\mathrm{cm})$ a cinco centímetros acima do ponto de

Tabela 1. Condições meteorológicas observadas durante a condução do experimento(1).

\begin{tabular}{|c|c|c|c|c|c|c|c|c|c|c|c|c|c|}
\hline Ano & Janeiro & Fevereiro & Março & Abril & Maio & Junho & Julho & Agosto & Setembro & Outubro & Novembro & Dezembro & $\begin{array}{l}\text { Média/ } \\
\text { Soma }^{(2)} \\
\end{array}$ \\
\hline & \multicolumn{13}{|c|}{ Média das temperaturas máximas mensais $\left({ }^{\circ} \mathrm{C}\right)$} \\
\hline 1996 & 22,1 & 22,5 & 21,1 & 19,2 & 16,9 & 12,6 & 15,8 & 17,4 & 17,0 & 16,9 & 19,6 & 22,0 & 18,6 \\
\hline 1997 & 22,2 & 21,6 & 20,2 & 18,0 & 15,5 & 14,3 & 14,7 & 14,4 & 15,2 & 17,8 & 19,8 & 21,2 & 17,9 \\
\hline 1998 & 22,8 & 22,2 & 22,4 & 16,9 & 13,9 & 13,8 & 13,9 & 17,2 & 17,8 & 16,2 & 18,5 & 21,7 & 18,1 \\
\hline 1999 & - & - & - & - & - & - & - & - & - & - & - & - & - \\
\hline 2000 & 22,5 & 21,4 & 20,2 & 19,3 & 14,8 & 16,0 & 12,3 & 16,2 & 16,1 & 19,6 & 20,3 & 22,3 & 18,4 \\
\hline 2001 & 22,8 & 23,3 & 22,7 & 19,8 & 14,4 & 14,9 & 15,7 & 18,4 & 15,5 & 18,9 & 21,4 & 21,3 & 19,1 \\
\hline 2002 & 22,1 & 21,3 & 23,8 & 20,2 & 17,2 & 15,3 & 14,5 & 17,4 & 15,6 & 20,4 & 21,0 & 22,3 & 19,3 \\
\hline 2003 & 22,8 & 23,7 & 21,8 & 18,6 & 15,7 & 16,1 & 16,2 & 15,0 & 17,9 & 19,7 & 20,1 & 20,5 & 19,0 \\
\hline 2004 & 22,2 & 21,4 & 21,3 & 20,1 & 13,7 & 16,0 & 13,3 & 17,2 & 19,0 & 18,4 & 19,9 & 20,5 & 18,6 \\
\hline 2005 & 23,9 & 22,4 & 22,5 & 18,1 & 17,3 & 16,6 & 13,5 & 17,8 & 13,1 & 18,2 & 20,7 & 21,1 & 18,8 \\
\hline 2006 & 23,6 & 22,9 & 22,4 & 18,7 & 13,9 & 14,4 & 16,7 & 16,6 & 16,4 & 20,7 & 18,9 & 23,6 & 19,1 \\
\hline \multirow[t]{2}{*}{2007} & 23,1 & 20,2 & 23,4 & 20,2 & 13,4 & 15,6 & 13,3 & 16,7 & 18,8 & 20,2 & 19,6 & 23,2 & 19,0 \\
\hline & \multicolumn{13}{|c|}{ Média das temperaturas mínimas mensais $\left({ }^{\circ} \mathrm{C}\right)$} \\
\hline 1996 & 13,2 & 13,4 & 12,1 & 10,7 & 7,3 & 4,0 & 2,3 & 7,8 & 6,2 & 9,1 & 10,5 & 13,5 & 9,2 \\
\hline 1997 & 14,0 & 15,0 & 11,5 & 9,2 & 7,9 & 5,6 & 7,6 & 8,5 & 7,8 & 9,8 & 11,7 & 13,6 & 10,2 \\
\hline 1998 & 14,1 & 13,6 & 12,4 & 10,3 & 7,3 & 5,2 & 6,5 & 7,6 & 7,4 & 9,2 & 9,7 & 11,9 & 9,6 \\
\hline 1999 & 13,5 & 13,8 & 13,8 & 9,4 & 5,8 & 5,3 & 5,3 & 6,3 & 7,1 & 7,3 & 8,1 & 11,9 & 9,0 \\
\hline 2000 & 13,6 & 12,9 & 11,6 & 10,5 & 6,6 & 8,3 & 2,4 & 5,7 & 6,7 & 10,7 & 10,6 & 12,4 & 9,3 \\
\hline 2001 & 14,2 & 15,4 & 14,3 & 12,4 & 6,9 & 6,8 & 6,1 & 9,8 & 8,1 & 10,6 & 11,6 & 11,7 & 10,7 \\
\hline 2002 & 13,4 & 12,0 & 15,3 & 12,7 & 9,7 & 8,1 & 6,0 & 8,7 & 6,0 & 11,7 & 11,3 & 13,3 & 10,7 \\
\hline 2003 & 17,4 & 15,4 & 13,4 & 10,0 & 7,2 & 9,1 & 8,2 & 4,2 & 7,0 & 9,4 & 10,4 & 11,6 & 10,3 \\
\hline 2004 & 12,8 & 11,8 & 11,5 & 11,8 & 6,3 & 7,9 & 5,0 & 6,6 & 10,1 & 7,5 & 10,5 & 11,2 & 9,4 \\
\hline 2005 & 13,1 & 12,3 & 12,6 & 10,8 & 8,6 & 9,3 & 5,2 & 8,6 & 5,5 & 10,2 & 10,3 & 10,8 & 9,8 \\
\hline 2006 & 14,1 & 13,9 & 13,1 & 9,1 & 5,7 & 7,4 & 8,6 & 7,2 & 6,7 & 10,4 & 10,0 & 13,8 & 10,0 \\
\hline \multirow[t]{2}{*}{2007} & 13,7 & 12,0 & 14,9 & 11,7 & 6,1 & 7,7 & 4,2 & 6,0 & 9,7 & 11,0 & 9,6 & 12,8 & 10,0 \\
\hline & \multicolumn{13}{|c|}{ Precipitação total mensal (mm) } \\
\hline 1996 & 296,1 & 117,0 & 199,8 & 60,6 & 44,9 & 145,4 & 152,7 & 191,0 & 159,2 & 158,2 & 53,5 & 121,0 & $1.699,4$ \\
\hline 1997 & 245,4 & 249,0 & 37,3 & 40,6 & 50,9 & 107,4 & 188,5 & 230,4 & 154,2 & 354,1 & 287,9 & 191,2 & $2.136,9$ \\
\hline 1998 & 265,8 & 360,0 & 163,9 & 232,8 & 132,9 & 152,3 & 153,6 & 224,6 & 253,5 & 94,0 & 92,3 & 122,6 & $2.248,3$ \\
\hline 1999 & 156,2 & 146,5 & 72,7 & 102,9 & 121,4 & 67,6 & $16,2,6$ & 38,3 & 95,3 & 127,7 & 138,3 & 121,4 & $1.188,3$ \\
\hline 2000 & 159,5 & 159,2 & 161,7 & 135,7 & 119,4 & 115,0 & 142,4 & 84,8 & 188,2 & 281,5 & 113,0 & 150,2 & $1.810,6$ \\
\hline 2001 & 385,5 & 199,4 & 88,1 & 154,8 & 201,7 & 137,5 & 236,0 & 60,9 & 226,3 & 197,2 & 190,9 & 130,8 & $2.209,1$ \\
\hline 2002 & 146,3 & 50,4 & 136,4 & 111,1 & 113,5 & 223,5 & 136,7 & 143,1 & 204,5 & 265,8 & 259,7 & 186,8 & $1.977,8$ \\
\hline 2003 & 118,3 & 227,0 & 129,3 & 103,2 & 85,0 & 102,9 & 70,8 & 466,0 & 82,5 & 131,7 & 126,8 & 339,0 & $1.982,5$ \\
\hline 2004 & 78,6 & 53,4 & 128,7 & 91,6 & 88,4 & 77,7 & 126,1 & 39,5 & 84,6 & 88,5 & 84,2 & 39,0 & 980,3 \\
\hline 2005 & 89,4 & 131,2 & 165,3 & 91,1 & 93,1 & 85,8 & 89,7 & 170,8 & 136,1 & 268,4 & 97,2 & 114,8 & $1.532,9$ \\
\hline 2006 & 155,2 & 54,2 & 41,3 & 53,4 & 51,0 & 120,7 & 129,7 & 199,1 & 85,3 & 102,0 & 266,8 & 122,6 & $1.381,3$ \\
\hline 2007 & 146,4 & 160,4 & 227,8 & 57,0 & 59,2 & 21,1 & 337,2 & 156,1 & 248,5 & 201,7 & 180,5 & 160,2 & $1.956,1$ \\
\hline
\end{tabular}

${ }^{(1)}$ Dados obtidos da estação meteorológica localizada na Estação Experimental de São Joaquim, SC. (2)Média, para temperaturas, e soma, para precipitação. 
enxertia, e expressa como área da seção transversal do tronco (ASTT), calculada conforme Pasa et al. (2012).

As análises estatísticas foram realizadas com o programa R (R Core Team, 2014) e o pacote ExpDes (Ferreira et al., 2013). Os dados foram submetidos à comparação de médias pelo teste de Duncan, a 5\% de probabilidade.

\section{Resultados e Discussão}

Com a cultivar Royal Gala, os porta-enxertos interagiram significativamente com a densidade de plantio, quanto à produção por planta, nas safras de 2005 e 2007 (Tabela 2). Em 2005, com o porta-enxerto M9, a maior produção por planta foi observada com as densidades de plantio de 1.667 e 2.500 plantas ha $^{-1}$. Com o porta-enxerto M26, no entanto, a maior produção por planta foi observada apenas na densidade de plantio de 1.667 plantas ha ${ }^{-1}$. Comportamentos semelhantes foram observados em 2007. Na comparação entre porta-enxertos, diferenças significativas foram observadas apenas quanto à densidade 1.667 plantas ha $^{-1}$, nas safras 2005 e 2007, em que as plantas do porta-enxerto M26 foram mais produtivas do que as do M9.

A interação entre densidades de plantio e porta-enxertos provavelmente decorreu dos diferentes graus de vigor induzidos por estes, que afetam a capacidade das plantas de preencher o espaço destinado à produção, na linha de plantio. A maior produção de M26, à menor densidade de plantio, evidencia justamente isso, uma vez que ele é mais vigoroso do que M9 (Jackson, 2003). Larsen et al. (1992) observaram que a produção por planta, ao longo dos anos, em macieiras 'Delicious' e 'Golden Delicious', tende a aumentar nos porta-enxertos vigorosos e diminuir nos porta-enxertos menos vigorosos. O mesmo conceito pode ser utilizado para explicar as diferenças entre as diferentes densidades de plantio, para cada porta-enxerto, uma vez que plantas sobre M9, à menor densidade de plantio, não foram mais produtivas do que no espaçamento intermediário, ou seja, as plantas não foram capazes de preencher todo o espaço destinado à produção na linha de plantio. No entanto, com M26 observou-se aumento gradual da produção por planta com a redução da densidade de plantio.

Ainda com relação à cultivar Royal Gala, na safra de 1998, não se observaram diferenças significativas entre os tratamentos, quanto à produção por planta, enquanto, nas demais safras, observou-se efeito isolado dos fatores densidade de plantio e porta-enxerto, uma vez que a interação não foi significativa (Tabela 2). Em 1999 e 2001, observou-se maior produção por planta com as densidades de 1.667 e 2.500 plantas ha-1 ${ }^{-1}$ No entanto, em 2000, 2002, 2003, 2004,

Tabela 2. Produção por planta (kg), nas safras de 1998 a 2007, e produção acumulada, em macieiras 'Royal Gala' e 'Fuji', a diferentes densidades de plantio, com diferentes porta-enxertos ${ }^{(1)}$.

\begin{tabular}{|c|c|c|c|c|c|c|c|c|c|c|c|c|c|}
\hline \multirow{2}{*}{$\begin{array}{l}\text { Fator de } \\
\text { variação }\end{array}$} & \multirow[t]{2}{*}{1998} & \multirow[t]{2}{*}{1999} & \multirow[t]{2}{*}{2000} & \multirow[t]{2}{*}{2001} & \multirow[t]{2}{*}{2002} & \multirow[t]{2}{*}{2003} & \multirow[t]{2}{*}{2004} & \multicolumn{2}{|c|}{2005} & \multirow[t]{2}{*}{2006} & \multicolumn{2}{|c|}{2007} & \multirow[t]{2}{*}{ Acumulada } \\
\hline & & & & & & & & M9 & M26 & & M9 & M26 & \\
\hline & \multicolumn{13}{|c|}{ Cultivar Royal Gala } \\
\hline \multicolumn{14}{|l|}{ Densidade } \\
\hline 5.000 plantas $\mathrm{ha}^{-1}$ & 0,5 & $6,0 \mathrm{~b}$ & $10,0 \mathrm{c}$ & $11,7 \mathrm{~b}$ & $10,3 \mathrm{c}$ & $13,7 \mathrm{c}$ & $11,5 \mathrm{c}$ & $8,6 \mathrm{bA}$ & $9,5 \mathrm{cA}$ & $12,6 \mathrm{c}$ & $16,2 \mathrm{bA}$ & $18,7 \mathrm{bA}$ & $102,7 \mathrm{c}$ \\
\hline 2.500 plantas ha $^{-1}$ & 0,4 & $8,2 \mathrm{a}$ & $13,6 b$ & $17,9 \mathrm{a}$ & $16,3 b$ & $21,2 b$ & $17,5 b$ & $15,5 \mathrm{aA}$ & $13,2 \mathrm{bA}$ & $16,8 b$ & $27,2 \mathrm{aA}$ & $22,9 \mathrm{bA}$ & $151,3 b$ \\
\hline 1.667 plantas ha ${ }^{-1}$ & 0,4 & $8,5 \mathrm{a}$ & $16,5 \mathrm{a}$ & $21,3 \mathrm{a}$ & $19,3 \mathrm{a}$ & $25,5 \mathrm{a}$ & $23,4 \mathrm{a}$ & $17,3 \mathrm{aB}$ & $20,2 \mathrm{aA}$ & $23,5 \mathrm{a}$ & $29,8 \mathrm{aB}$ & $38,2 \mathrm{aA}$ & $191,2 \mathrm{a}$ \\
\hline \multicolumn{14}{|l|}{ Porta-enxerto } \\
\hline M9 & 0,5 & 6,8 & $11,3 b$ & 16,2 & 15,1 & 19,4 & 16,8 & - & - & 17,2 & - & - & 141,4 \\
\hline M26 & 0,3 & 8,3 & $15,5 \mathrm{a}$ & 17,7 & 15,5 & 20,8 & 18,2 & - & - & 18,1 & - & - & 155,4 \\
\hline & \multicolumn{13}{|c|}{ Cultivar Fuji } \\
\hline \multicolumn{14}{|l|}{ Densidade } \\
\hline 5.000 plantas $\mathrm{ha}^{-1}$ & 0,5 & 7,1 & $13,4 \mathrm{c}$ & $12,1 \mathrm{~b}$ & $12,8 \mathrm{c}$ & $20,3 b$ & $14,9 \mathrm{c}$ & \multicolumn{2}{|c|}{$14,5 \mathrm{c}$} & $22,0 \mathrm{c}$ & \multicolumn{2}{|c|}{$28,4 \mathrm{c}$} & $145,9 \mathrm{c}$ \\
\hline 2.500 plantas $\mathrm{ha}^{-1}$ & 0,6 & 8,8 & $21,3 b$ & $18,5 \mathrm{a}$ & $19,1 b$ & $29,5 \mathrm{a}$ & $25,7 b$ & \multicolumn{2}{|c|}{$24,7 b$} & $31,5 b$ & \multicolumn{2}{|c|}{$43,0 \mathrm{~b}$} & $222,6 b$ \\
\hline 1.667 plantas ha ${ }^{-1}$ & 0,6 & 9,1 & $27,4 \mathrm{a}$ & $21,9 \mathrm{a}$ & $25,6 \mathrm{a}$ & $35,2 \mathrm{a}$ & $34,6 \mathrm{a}$ & \multicolumn{2}{|c|}{$33,5 \mathrm{a}$} & $38,6 \mathrm{a}$ & \multicolumn{2}{|c|}{$53,5 \mathrm{a}$} & $279,8 \mathrm{a}$ \\
\hline \multicolumn{14}{|l|}{ Porta-enxerto } \\
\hline M9 & 0,6 & $7,5 \mathrm{~b}$ & 19,1 & $14,6 b$ & 19,0 & $25,7 b$ & 24,0 & \multicolumn{2}{|c|}{23,1} & $25,4 b$ & \multicolumn{2}{|c|}{$34,0 \mathrm{~b}$} & $193,1 b$ \\
\hline M26 & 0,5 & $9,1 \mathrm{a}$ & 22,2 & $20,4 \mathrm{a}$ & 19,3 & $30,9 a$ & 26,1 & \multicolumn{2}{|c|}{25,3} & $35,9 \mathrm{a}$ & \multicolumn{2}{|c|}{$49,2 \mathrm{a}$} & $239,1 \mathrm{a}$ \\
\hline
\end{tabular}

${ }^{(1)}$ Médias seguidas de letras iguais, minúsculas nas colunas e maiúsculas nas linhas, não diferem pelo teste de Duncan, a 5\% de probabilidade. 
2006 e na produção acumulada, os maiores valores por planta foram obtidos com a densidade de 1.667 plantas ha $^{-1}$, e os menores, com 5.000 plantas ha ${ }^{-1}$. Quanto aos porta-enxertos, houve diferença significativa entre eles apenas em 2000, em que a produção por planta no porta-enxerto M26 foi maior do que aquela em M9.

$\mathrm{Na}$ cultivar Fuji, não se constataram interações significativas entre densidades de plantio e porta-enxertos, quanto à variável produção por planta, em nenhum dos anos de avaliação, tendo-se observado apenas efeitos significativos de fatores isolados (Tabela 2). O fator densidade de plantio apresentou influência significativa sobre os resultados, em todos os anos avaliados, exceto em 1998 e 1999, e na produção acumulada. Em 2001 e 2003, as densidades de 1.667 e 2.500 plantas ha $^{-1}$ foram superiores à de 5.000 plantas $\mathrm{ha}^{-1}$. Nos demais anos, a menor densidade de plantio foi superior às demais. Quanto aos porta-enxertos, a produção por planta foi maior com M26 em 1999, 2001, 2003, 2006, 2007 e na produção acumulada. Nos demais anos, não se observaram diferenças significativas.

Quanto à variável número de frutos por planta, observou-se interação entre os fatores apenas em 2005, para a 'Royal Gala', e em 1999, para a 'Fuji' (Tabela 3). Com a 'Royal Gala', em 2005, o menor número de frutos por planta foi observado à densidade de 5.000 plantas $\mathrm{ha}^{-1}$, independentemente do porta-enxerto; porém, com M26, o número de frutos por planta, à densidade de 2.500 plantas ha ${ }^{-1}$, foi intermediário. $\mathrm{O}$ número de frutos por planta, à densidade de 1.667 plantas ha $^{-1}$, foi maior com M26. Em 2000 e 2001, as densidades de 1.667 e 2.500 plantas ha ${ }^{-1}$ resultaram em maior número de frutos. Em 2002, 2003, 2004, 2006, 2007 e no resultado acumulado, o número de frutos foi maior com 1.667 plantas ha $^{-1}$, intermediário com 2.500 plantas ha $^{-1}$ e menor com 5.000 plantas ha-1.

Com a 'Fuji', em 1999, não se observaram diferenças entre densidades de plantio com o M9. No entanto, com o M26, o número de frutos, à densidade de 1.667 plantas $\mathrm{ha}^{-1}$, foi maior do que à densidade de 5.000 plantas ha $^{-1}$. Em 2000, 2001 e 2003, observou-se maior número de frutos, às densidades de 1.667 e 2.500 plantas ha $^{-1}$. Todavia, em 2002, 2004, 2005, 2006, 2007 e no resultado acumulado, o número de frutos foi maior à densidade de 1.667 plantas ha $^{-1}$, intermediário à de 2.500 plantas ha $^{-1} \mathrm{e}$ menor à de 5.000 plantas ha ${ }^{-1}$ (Tabela 3 ).

Diferenças entre os porta-enxertos foram observadas em 2000, 2002, 2003 e no resultado acumulado, com a 'Royal Gala' e, em 2000, 2001, 2003, 2006, 2007 e no resultado acumulado, com a 'Fuji' (Tabela 3), em que o maior número de frutos foi observado com o M26.

Tabela 3. Número de frutos por planta, nas safras de 1998 a 2007, e número médio de frutos em macieiras 'Royal Gala' e 'Fuji', a diferentes densidades de plantio, com diferentes porta-enxertos ${ }^{(1)}$.

\begin{tabular}{|c|c|c|c|c|c|c|c|c|c|c|c|c|c|}
\hline \multirow{2}{*}{$\begin{array}{l}\text { Fator de } \\
\text { variação }\end{array}$} & \multirow[t]{2}{*}{1998} & \multicolumn{2}{|c|}{1999} & \multirow[t]{2}{*}{2000} & \multirow[t]{2}{*}{2001} & \multirow[t]{2}{*}{2002} & \multirow[t]{2}{*}{2003} & \multirow[t]{2}{*}{2004} & \multicolumn{2}{|c|}{2005} & \multirow[t]{2}{*}{2006} & \multirow[t]{2}{*}{2007} & \multirow[t]{2}{*}{ Média } \\
\hline & & M9 & M26 & & & & & & M9 & M26 & & & \\
\hline & & \multicolumn{12}{|c|}{ Cultivar Royal Gala } \\
\hline \multicolumn{14}{|l|}{ Densidade } \\
\hline 5.000 plantas ha ${ }^{-1}$ & 4,1 & \multicolumn{2}{|c|}{45,2} & $77,1 \mathrm{~b}$ & $87,0 \mathrm{~b}$ & $78,0 \mathrm{c}$ & $103,3 \mathrm{c}$ & $95,5 \mathrm{c}$ & $67,2 \mathrm{bA}$ & $76,8 \mathrm{cA}$ & $87,5 \mathrm{c}$ & $142,2 \mathrm{c}$ & $791,9 \mathrm{c}$ \\
\hline 2.500 plantas $\mathrm{ha}^{-1}$ & 3,3 & \multicolumn{2}{|c|}{57,1} & $101,7 \mathrm{a}$ & $120,8 \mathrm{a}$ & $116,1 b$ & $154,0 \mathrm{~b}$ & $138,1 b$ & $117,8 \mathrm{aA}$ & $102,4 \mathrm{bA}$ & $111,7 b$ & $198,1 b$ & $1.110,9 b$ \\
\hline 1.667 plantas ha-1 & 2,8 & \multicolumn{2}{|c|}{57,1} & $117,0 \mathrm{a}$ & $140,0 \mathrm{a}$ & $138,1 \mathrm{a}$ & $177,0 \mathrm{a}$ & $179,5 \mathrm{a}$ & $132,9 \mathrm{aB}$ & $156,7 \mathrm{aA}$ & $150,8 \mathrm{a}$ & $250,1 \mathrm{a}$ & $1.357,1 \mathrm{a}$ \\
\hline \multicolumn{14}{|l|}{ Porta-enxerto } \\
\hline M9 & 3,8 & \multicolumn{2}{|c|}{47,3} & $79,8 b$ & 111,9 & $100,4 b$ & $133,8 b$ & 128,3 & - & - & 113,2 & 185,1 & $1.009,6 \mathrm{~b}$ \\
\hline M26 & 3,0 & \multicolumn{2}{|c|}{59,0} & $117,3 \mathrm{a}$ & 120,0 & $121,0 \mathrm{a}$ & $155,7 \mathrm{a}$ & 147,0 & - & - & 120,2 & 208,5 & $1.163,7 \mathrm{a}$ \\
\hline & & \multicolumn{12}{|c|}{ Cultivar Fuji } \\
\hline \multicolumn{14}{|l|}{ Densidade } \\
\hline 5.000 plantas $\mathrm{ha}^{-1}$ & 2,6 & $37,6 \mathrm{aA}$ & $39,3 \mathrm{bA}$ & $74,7 \mathrm{~b}$ & $64,7 b$ & $78,1 \mathrm{c}$ & $130,5 b$ & $102,9 \mathrm{c}$ & \multicolumn{2}{|c|}{$87,3 \mathrm{c}$} & $130,8 \mathrm{c}$ & $168,4 \mathrm{c}$ & $878,4 \mathrm{c}$ \\
\hline 2.500 plantas $\mathrm{ha}^{-1}$ & 3,4 & $45,2 \mathrm{aA}$ & $47,3 \mathrm{abA}$ & $104,3 \mathrm{a}$ & $95,4 \mathrm{a}$ & $106,9 b$ & $179,2 \mathrm{a}$ & $171,6 b$ & \multicolumn{2}{|c|}{$146,6 b$} & $185,9 b$ & $238,9 b$ & $1.278,4 \mathrm{~b}$ \\
\hline 1.667 plantas ha-1 & 2,9 & $38,0 \mathrm{aB}$ & $56,6 \mathrm{aA}$ & $128,1 \mathrm{a}$ & $111,4 \mathrm{a}$ & $147,7 \mathrm{a}$ & $211,0 \mathrm{a}$ & $224,7 \mathrm{a}$ & \multicolumn{2}{|c|}{$190,7 \mathrm{a}$} & $221,3 \mathrm{a}$ & $297,5 \mathrm{a}$ & $1.582,5 \mathrm{a}$ \\
\hline \multicolumn{14}{|l|}{ Porta-enxerto } \\
\hline M9 & 2,8 & - & - & $90,9 b$ & $73,8 b$ & 104,4 & $154,1 b$ & 157,1 & \multicolumn{2}{|c|}{133,9} & $151,2 \mathrm{~b}$ & $198,8 b$ & $1.107,1 \mathrm{~b}$ \\
\hline M26 & 3,2 & - & - & $113,8 \mathrm{a}$ & $107,3 \mathrm{a}$ & 117,4 & $193,0 \mathrm{a}$ & 175,7 & \multicolumn{2}{|c|}{149,1} & $207,5 \mathrm{a}$ & $271,1 \mathrm{a}$ & $1.385,7 \mathrm{a}$ \\
\hline
\end{tabular}

${ }^{(1)}$ Médias seguidas de letras iguais, minúsculas nas colunas e maiúsculas nas linhas, não diferem pelo teste de Duncan, a 5\% de probabilidade. 
Al-Hinai \& Roper (2004) também relataram maior número de frutos com esse porta-enxerto.

Em geral, as variáveis produção e número de frutos por planta apresentaram relação inversa com a densidade de plantio. Efeito semelhante também foi relatado quanto a macieiras 'Red Spur' (Pramanick et al., 2012), 'Royal Gala' (Hampson et al., 2002), 'Golden Delicious', 'Braeburn' e 'Fuji' (Eccher \& Granelli, 2006), bem como em pereiras 'Santa Maria' (Pasa et al., 2015). Possivelmente, esse efeito está relacionado à redução do sistema radicular das plantas às maiores densidades de plantio, conforme observado em macieiras 'Pink Lady' (Lo Bianco et al., 2003) e em pereiras 'William's' (Policarpo et al., 2006). Nessa situação, as plantas mais adensadas teriam desvantagem na competição por recursos do solo, em comparação às menos adensadas, o que afetaria a sua capacidade produtiva.

Para a variável produtividade, houve interação entre os fatores apenas em 2005, com a 'Royal Gala' (Tabela 4). Para o porta-enxerto M9, a maior produtividade foi observada às densidades de 2.500 e 5.000 plantas ha $^{-1}$, enquanto para o $\mathrm{M} 26$, a maior produtividade foi obtida à densidade de 5.000 plantas ha $^{-1}$. Quanto às demais variáveis, apenas os fatores principais foram significativos (densidade de plantio e porta-enxerto). A densidade de 5.000 plantas $\mathrm{ha}^{-1}$ apresentou produtividade superior à das demais em 1998, 2000, 2004, 2006 e 2007; enquanto as de 1.667 e 2.500 plantas ha ${ }^{-1}$ não diferiram entre si. Efeito semelhante foi observado em 1999, 2001, 2002, 2003, e na produtividade acumulada, em que a produtividade foi superior quando a densidade foi de 5.000 plantas ha $^{-1}$; tendo-se observado, no entanto, maior produtividade com 2.500 plantas $\mathrm{ha}^{-1}$ do que com 1.667 plantas ha ${ }^{-1}$. Diferenças entre os porta-enxertos foram observadas apenas em 2000 e na produtividade acumulada, e o M26 foi mais produtivo do que o M9.

Para a cultivar Fuji, constatou-se efeito apenas dos fatores principais sobre a produtividade (Tabela 4). A maior produtividade foi observada à densidade de 5.000 plantas ha $^{-1}$, em todos os anos de avaliação e no resultado acumulado, exceto em 2004, quando não diferiu significativamente da observada com 2.500 plantas $\mathrm{ha}^{-1}$. Os porta-enxertos diferiram significativamente em 2001, 2003, 2007 e quanto à produtividade acumulada, com maior produtividade para o M26.

Com o aumento da densidade de plantio, a menor produção por planta foi compensada pelo maior número de plantas por hectare e, ao final de 10 safras, o plantio mais adensado resultou, claramente, na maior produtividade. Esse comportamento também foi observado em macieiras 'Red Spur' (Pramanick et al., 2012), 'Royal Gala' (Hampson et al., 2004a), 'Golden Delicious', 'Braeburn' e 'Fuji' (Eccher \& Granelli,

Tabela 4. Produtividade ( $\left.\mathrm{Mg} \mathrm{ha}^{-1}\right)$, nas safras de 1998 a 2007, e produtividade acumulada de macieiras 'Royal Gala' e 'Fuji', a diferentes densidades de plantio, com diferentes porta-enxertos ${ }^{(1)}$.

\begin{tabular}{|c|c|c|c|c|c|c|c|c|c|c|c|c|}
\hline \multirow{2}{*}{$\begin{array}{l}\text { Fator de } \\
\text { variação }\end{array}$} & \multirow[t]{2}{*}{1998} & \multirow[t]{2}{*}{1999} & \multirow[t]{2}{*}{2000} & \multirow[t]{2}{*}{2001} & \multirow[t]{2}{*}{2002} & \multirow[t]{2}{*}{2003} & \multirow[t]{2}{*}{2004} & \multicolumn{2}{|c|}{2005} & \multirow[t]{2}{*}{2006} & \multirow[t]{2}{*}{2007} & \multirow[t]{2}{*}{ Acumulada } \\
\hline & & & & & & & & M9 & M26 & & & \\
\hline & \multicolumn{12}{|c|}{ Cultivar Royal Gala } \\
\hline \multicolumn{13}{|l|}{ Densidade } \\
\hline 5.000 plantas $\mathrm{ha}^{-1}$ & $2,3 a$ & $28,3 \mathrm{a}$ & $49,9 \mathrm{a}$ & $58,7 \mathrm{a}$ & $51,4 \mathrm{a}$ & $68,4 \mathrm{a}$ & $57,5 \mathrm{a}$ & $43,1 \mathrm{aA}$ & $47,2 \mathrm{aA}$ & $63,0 \mathrm{a}$ & $87,2 \mathrm{a}$ & $511,9 \mathrm{a}$ \\
\hline 2.500 plantas ha $^{-1}$ & $1,0 \mathrm{~b}$ & $20,4 b$ & $33,9 b$ & $44,7 b$ & $41,3 b$ & $53,0 \mathrm{~b}$ & $43,8 b$ & $38,7 \mathrm{aA}$ & $33,0 \mathrm{bA}$ & $42,1 b$ & $62,7 \mathrm{~b}$ & $378,7 \mathrm{~b}$ \\
\hline 1.667 plantas ha $^{-1}$ & $0,6 \mathrm{~b}$ & $14,2 \mathrm{c}$ & $27,6 b$ & $35,5 \mathrm{c}$ & $32,1 \mathrm{c}$ & $42,6 \mathrm{c}$ & $39,1 \mathrm{~b}$ & $28,9 \mathrm{bA}$ & $33,6 \mathrm{bA}$ & $39,1 \mathrm{~b}$ & $56,7 \mathrm{~b}$ & $318,7 \mathrm{c}$ \\
\hline \multicolumn{13}{|l|}{ Porta-enxerto } \\
\hline M9 & 1,4 & 18,9 & $31,3 b$ & 43,9 & 40,3 & 51,9 & 44,6 & - & - & 46,9 & 66,3 & $382,4 b$ \\
\hline M26 & 1,2 & 23,1 & $43,0 \mathrm{a}$ & 48,7 & 42,9 & 57,3 & 49,0 & - & - & 49,2 & 71,4 & $423,8 \mathrm{a}$ \\
\hline & \multicolumn{12}{|c|}{ Cultivar Fuji } \\
\hline \multicolumn{13}{|l|}{ Densidade } \\
\hline 5.000 plantas $\mathrm{ha}^{-1}$ & $2,5 \mathrm{a}$ & $35,5 \mathrm{a}$ & $66,8 \mathrm{a}$ & $60,5 \mathrm{a}$ & $64,2 \mathrm{a}$ & $94,7 \mathrm{a}$ & $74,4 \mathrm{a}$ & \multicolumn{2}{|c|}{65,9} & 91,6 & $136,2 \mathrm{a}$ & $692,4 a$ \\
\hline 2.500 plantas ha $^{-1}$ & $1,5 b$ & $21,9 b$ & $53,3 b$ & $46,4 b$ & $47,7 \mathrm{~b}$ & $73,7 b$ & $64,3 \mathrm{ab}$ & \multicolumn{2}{|c|}{61,7} & 78,7 & $107,5 \mathrm{~b}$ & $556,6 \mathrm{~b}$ \\
\hline 1.667 plantas $\mathrm{ha}^{-1}$ & $1,1 \mathrm{~b}$ & $17,5 b$ & $45,6 \mathrm{~b}$ & $36,5 b$ & $42,7 \mathrm{~b}$ & $58,6 \mathrm{c}$ & $57,7 \mathrm{~b}$ & \multicolumn{2}{|c|}{59,8} & 68,0 & $89,1 \mathrm{~b}$ & $476,5 b$ \\
\hline \multicolumn{13}{|l|}{ Porta-enxerto } \\
\hline M9 & 1,7 & 22,3 & 51,3 & $40,7 \mathrm{~b}$ & 51,1 & $69,5 b$ & 63,2 & \multicolumn{2}{|c|}{63,2} & 72,2 & $91,7 \mathrm{~b}$ & $527,0 \mathrm{~b}$ \\
\hline M26 & 1,7 & 27,6 & 59,2 & $54,9 \mathrm{a}$ & 52,0 & $81,8 \mathrm{a}$ & 67,7 & \multicolumn{2}{|c|}{61,8} & 86,6 & $130,2 \mathrm{a}$ & $623,3 \mathrm{a}$ \\
\hline
\end{tabular}

(1)Médias seguidas de letras iguais, minúsculas nas colunas e maiúsculas nas linhas, não diferem pelo teste de Duncan, a $5 \%$ de probabilidade. 
2006). A melhor interceptação de luz proporcionada pelos plantios mais adensados, provavelmente, foi a principal razão para as maiores produtividades observadas (Verheij \& Verwer, 1973). Jung \& Choi, (2010) observaram relação positiva e linear da densidade de plantio com a produtividade.

A maior produtividade acumulada induzida pelo porta-enxerto M26, em ambas as cultivares, provavelmente, é resultante de seu maior vigor em relação a M9 (Rom \& Carlson, 1987; Jackson, 2003). Esse maior vigor possibilita o preenchimento mais rápido do espaço nas linhas de plantio. Resultados semelhantes foram observados em macieiras 'Cripps Pink' e 'Cripps Red' (James \& Middleton, 2011). Embora os porta-enxertos não tenham diferido significativamente, na maioria das safras avaliadas, a produtividade com M26, em geral, foi levemente superior à do M9, o que provavelmente levou às diferenças observadas quanto à produtividade acumulada.

Quanto à massa de fruto, apenas os fatores principais tiveram efeito significativo, em ambas as cultivares (Tabela 5). Na cultivar Royal Gala, a densidade de plantio foi significativa para os anos 2001, 2003, 2004, 2006 e para massa média dos frutos. Em 2001, 2003 e 2006, à densidade de plantio de 1.667 plantas ha- ${ }^{-1}$, os frutos apresentaram maior massa do que aqueles à densidade de 5.000 plantas ha' ${ }^{-1}$. Em 2004, para 'Royal Gala', e em 2000, 2001 e 2003, para 'Fuji', a massa de frutos à densidade de 5.000 plantas ha ${ }^{-1}$ foi inferior à observada nas demais densidades. A massa média dos frutos foi maior à densidade de plantio de 1.667 plantas $\mathrm{ha}^{-1}$, menor à de 5.000 plantas $\mathrm{ha}^{-1} \mathrm{e}$ intermediária à de 2.500 plantas ha ${ }^{-1}$, tanto para 'Royal Gala' quanto para 'Fuji'. Quanto aos porta-enxertos, diferenças significativas foram observadas quanto aos anos 2000, 2002, 2003, 2004 e quanto à massa média dos frutos, em 'Royal Gala', e, quanto a 2000, 2002, 2003 e 2007, na 'Fuji', em que os frutos enxertados sobre M9 foram maiores dos que os sobre M26.

A diminuição do tamanho de fruto tem sido comumente relacionada às maiores cargas de frutos em sistemas adensados (Robinson, 2011b). Porém, esse efeito está mais associado à maior carga de frutos por área do que por planta, pois as plantas mais adensadas apresentaram menor número de frutos por planta e menor massa. Resultados semelhantes foram observados por Pramanick et al. (2012), em macieiras 'Red Spur', e Pasa et al. (2015), em pereiras 'Rocha'. O efeito negativo do adensamento de plantio sobre a massa de fruto pode estar relacionado à maior competição por água e nutrientes em menores espaçamentos. Em macieiras 'Red Spur', as plantas à maior densidade de plantio apresentaram menor acúmulo foliar de N, P, K e Z (Pramanick et al., 2012).

A maior massa de fruto observada, na grande maioria dos tratamentos, com o M9 pode ser atribuída, em parte, ao menor número de frutos por

Tabela 5. Massa de fruto (g), nas safras de 1998 a 2007, e massa média de frutos de macieiras 'Royal Gala' e 'Fuji', a diferentes densidades de plantio, com diferentes porta-enxertos ${ }^{(1)}$.

\begin{tabular}{|c|c|c|c|c|c|c|c|c|c|c|c|}
\hline \multirow{2}{*}{$\begin{array}{l}\text { Fator de } \\
\text { variação }\end{array}$} & 1998 & 1999 & 2000 & 2001 & 2002 & 2003 & 2004 & 2005 & 2006 & 2007 & Média \\
\hline & \multicolumn{11}{|c|}{ Cultivar Royal Gala } \\
\hline \multicolumn{12}{|l|}{ Densidade } \\
\hline 5.000 plantas $\mathrm{ha}^{-1}$ & 108,0 & 136,1 & 131,2 & $137,6 \mathrm{~b}$ & 135,9 & $133,4 b$ & $122,4 b$ & 125,8 & $144,2 b$ & 122,6 & $129,7 \mathrm{c}$ \\
\hline 2.500 plantas ha ${ }^{-1}$ & 116,9 & 147,7 & 137,7 & $148,9 \mathrm{ab}$ & 142,2 & $137,5 \mathrm{ab}$ & $127,8 \mathrm{a}$ & 130,4 & $150,8 \mathrm{ab}$ & 128,0 & $136,8 b$ \\
\hline 1.667 plantas $\mathrm{ha}^{-1}$ & 125,0 & 150,1 & 141,0 & $152,6 \mathrm{a}$ & 142,2 & $145,2 \mathrm{a}$ & $131,8 \mathrm{a}$ & 129,8 & $155,3 \mathrm{a}$ & 135,4 & $140,8 \mathrm{a}$ \\
\hline \multicolumn{12}{|l|}{ Porta-enxerto } \\
\hline M9 & 116,6 & 144,4 & $141,0 \mathrm{a}$ & 145,7 & $152,0 \mathrm{a}$ & $144,2 \mathrm{a}$ & $130,9 \mathrm{a}$ & 130,4 & 151,3 & 131,5 & $138,8 \mathrm{a}$ \\
\hline M26 & 116,6 & 144,8 & $132,3 b$ & 147,0 & $128,2 \mathrm{~b}$ & $133,3 b$ & $123,7 \mathrm{~b}$ & 127,0 & 148,8 & 125,8 & $132,8 b$ \\
\hline & \multicolumn{11}{|c|}{ Cultivar Fuji } \\
\hline \multicolumn{12}{|l|}{ Densidade } \\
\hline 5.000 plantas ha ${ }^{-1}$ & 204,9 & 184,0 & $181,0 \mathrm{~b}$ & $187,0 \mathrm{~b}$ & 166,1 & $156,4 b$ & 148,1 & 168,0 & 168,4 & 166,3 & $173,0 \mathrm{~b}$ \\
\hline 2.500 plantas ha ${ }^{-1}$ & 197,7 & 189,9 & $209,6 \mathrm{a}$ & $195,9 \mathrm{a}$ & 180,2 & $166,3 \mathrm{a}$ & 152,7 & 171,5 & 169,9 & 180,6 & $181,4 \mathrm{a}$ \\
\hline 1.667 plantas ha ${ }^{-1}$ & 197,4 & 195,6 & $218,3 \mathrm{a}$ & $198,2 \mathrm{a}$ & 174,9 & $167,9 \mathrm{a}$ & 155,9 & 176,9 & 173,6 & 180,0 & $183,9 \mathrm{a}$ \\
\hline \multicolumn{12}{|l|}{ Porta-enxerto } \\
\hline M9 & 216,0 & 189,3 & $207,9 \mathrm{a}$ & 197,9 & $180,7 \mathrm{a}$ & $166,6 \mathrm{a}$ & 154,6 & 173,7 & 167,5 & $169,8 b$ & 182,4 \\
\hline M26 & 184,0 & 190,3 & $198,0 \mathrm{~b}$ & 189,5 & $166,8 \mathrm{~b}$ & $160,5 b$ & 149,9 & 170,5 & 173,7 & $181,5 \mathrm{a}$ & 176,5 \\
\hline
\end{tabular}

${ }^{(1)}$ Médias seguidas de letras iguais, nas colunas, não diferem pelo teste de Duncan, a 5\% de probabilidade. 
planta, geralmente observado com esse porta-enxerto (Tabela 5). Além disso, o M9 apresenta tendência de produzir frutos de maior calibre (Rom \& Carlson, 1987). Al-Hinai \& Roper (2004) não observaram influência do porta-enxerto sobre o tamanho de frutos de macieiras 'Gala'; porém, os autores relataram que a carga de frutos por planta influencia significativamente o tamanho de fruto.

A área da seção transversal do tronco foi influenciada significativamente apenas pelos fatores principais, independentemente da cultivar (Tabela 6). A área da seção transversal do tronco diferiu entre os tratamentos, em todos os anos de avaliação, com exceção de 1999, em que a densidade de 1.667 plantas $\mathrm{ha}^{-1}$ apresentou a maior área, e a de 5.000 plantas ha ${ }^{-1}$, a menor. Diferenças entre os porta-enxertos ocorreram apenas em 1999 e 2001, para 'Royal Gala', e em todos os anos para 'Fuji', em que M26 foi superior a M.9.

A redução da área da seção transversal do tronco, com o aumento da densidade de plantio, já foi relatada em macieiras 'Red Spur' (Pramanick et al., 2012), 'Royal Gala', 'Summerland McIntosh' (Hampson et al., 2004b), e em pereiras 'Rocha' (Pasa et al., 2015), 'Conference', 'Abbé Fétel' e 'Comice' (Musacchi et al., 2005). A maior área da seção transversal do

Tabela 6. Área da seção transversal do tronco $\mathrm{cm}^{-2}$, nas safras de 1999, 2001, 2004, 2005, 2006 e 2007, em macieiras 'Royal Gala' e 'Fuji', a diferentes densidades de plantio, com diferentes porta-enxertos ${ }^{(1)}$.

\begin{tabular}{lcccccc}
\hline $\begin{array}{l}\text { Fator de } \\
\text { variação }\end{array}$ & 1999 & 2001 & 2004 & 2005 & 2006 & 2007 \\
\hline \multicolumn{7}{c}{ Cultivar Royal Gala } \\
$\begin{array}{l}\text { Densidade } \\
\quad 5.000 \text { plantas ha }^{-1}\end{array}$ & 7,7 & $11,0 \mathrm{c}$ & $17,3 \mathrm{c}$ & $23,2 \mathrm{c}$ & $27,1 \mathrm{c}$ & $28,4 \mathrm{c}$ \\
2.500 plantas ha $^{-1}$ & 8,4 & $12,9 \mathrm{~b}$ & $22,7 \mathrm{~b}$ & $30,9 \mathrm{~b}$ & $34,6 \mathrm{~b}$ & $38,6 \mathrm{~b}$ \\
1.667 plantas ha $^{-1}$ & 9,0 & $15,6 \mathrm{a}$ & $29,5 \mathrm{a}$ & $37,0 \mathrm{a}$ & $43,3 \mathrm{a}$ & $45,1 \mathrm{a}$ \\
Porta-enxerto & & & & & & \\
M9 & $7,8 \mathrm{~b}$ & $12,2 \mathrm{~b}$ & 22,2 & 29,4 & 34,0 & 37,0 \\
M26 & $8,9 \mathrm{a}$ & $14,1 \mathrm{a}$ & 24,1 & 31,2 & 36,0 & 37,8 \\
\hline & & & Cultivar Fuji & & \\
Densidade & & & & & & \\
5.000 plantas ha $^{-1}$ & 10,2 & $16,7 \mathrm{~b}$ & $22,9 \mathrm{c}$ & $32,8 \mathrm{~b}$ & - & $47,2 \mathrm{c}$ \\
2.500 plantas ha $^{-1}$ & 12,6 & $21,7 \mathrm{a}$ & $34,7 \mathrm{~b}$ & $43,6 \mathrm{a}$ & - & $57,3 \mathrm{~b}$ \\
1.667 plantas ha $^{-1}$ & 13,1 & $21,8 \mathrm{a}$ & $44,7 \mathrm{a}$ & $49,8 \mathrm{a}$ & - & $67,7 \mathrm{a}$ \\
Porta-enxerto & & & & & & \\
$\quad$ M9 & $10,1 \mathrm{~b}$ & $15,9 \mathrm{~b}$ & $25,9 \mathrm{~b}$ & $34,2 \mathrm{~b}$ & - & $45,8 \mathrm{~b}$ \\
M26 & $13,8 \mathrm{a}$ & $24,1 \mathrm{a}$ & $42,3 \mathrm{a}$ & $49,9 \mathrm{a}$ & - & $69,0 \mathrm{a}$ \\
\hline
\end{tabular}

${ }^{(1)}$ Médias seguidas de letras iguais, nas colunas, não diferem entre si, pelo teste de Duncan, a $5 \%$ de probabilidade. tronco de M26, nos primeiros anos do pomar, pode ser explicada pelo fato de esse porta-enxerto ser semi-ananicante, ao passo que M9 é ananicante (Rom \& Carlson, 1987; Jackson, 2003).

\section{Conclusões}

1. O aumento da densidade de plantio incrementa a produtividade e diminui a produção, o número de frutos por planta e a massa dos frutos, em macieiras 'Royal Gala' e 'Fuji'.

2. O aumento da densidade de plantio reduz o vigor das plantas, expresso como área da seção transversal do tronco, em ambas as cultivares.

3. O porta-enxerto M26 favorece a obtenção de maior produtividade acumulada das macieiras avaliadas, enquanto o porta-enxerto M9 favorece a obtenção de frutos maiores.

\section{Referências}

A CULTURA da macieira. Florianópolis: Epagri, 2006. 743p.

AL-HINAI, Y.K.; ROPER, T.R. Rootstock effects on growth and quality of 'Gala' apples. HortScience, v.39, p.1231-1233, 2004.

ECCHER, T.; GRANELLI, G. Fruit quality and yield of different apple cultivars as affected by tree density. Acta Horticulturae, v.712, p.535-540, 2006. DOI: 10.17660/ActaHortic.2006.712.66.

FACHINELLO, J.C.; PASA, M. da S.; SCHMTIZ, J.D.; BETEMPS, D.L. Situação e perspectivas da fruticultura de clima temperado no Brasil. Revista Brasileira de Fruticultura, v.33, p.109-120, 2011.

FERREIRA, E.B.; CAVALCANTI, P.P.; NOGUEIRA, D.A. ExpDes: experimental designs package: version 1.1.2. 2013. Disponível em: <https://cran.r-project.org/web/packages/ExpDes/ index.html $>$. Acesso em: 4 maio 2015.

GJAMOVSKI, V.; KIPRIJANOVSKI, M. Influence of nine dwarfing apple rootstocks on vigour and productivity of apple cultivar 'Granny Smith'. Scientia Horticulturae, v.129, p.742-746, 2011. DOI: 10.1016/j.scienta.2011.05.032.

HAMPSON, C.R.; QUAMME, H.A.; BROWNLEE, R.T. Canopy growth, yield, and fruit quality of 'Royal Gala' apple trees grown for eight years in five tree training systems. HortScience, v.37, p.627-631, 2002.

HAMPSON, C.R.; QUAMME, H.A.; KAPPEL, F.; BROWNLEE, R.T. Varying density with constant rectangularity: II. Effects on apple tree yield, fruit size, and fruit color development in three training systems over ten years. HortScience, v.39, p.507-511, 2004a.

HAMPSON, C.R.; QUAMME, H.A.; KAPPEL, F.; BROWNLEE, R.T. Varying density with constant rectangularity: I. Effects on apple tree growth and light interception in three training systems over ten years. HortScience, v.39, p.501-506, 2004 b. 
INSTITUTO BRASILEIRO DE GEOGRAFIA E ESTATÍSTICA. Censo agropecuário 2013: lavoura permanente e temporária. Disponível em: <http://www.ibge.gov.br/estadosat/>. Acesso em: 1 jun. 2015.

JACKSON, J.E. Biology of apples and pears. Cambridge: Cambridge University Press, 2003. 501p. DOI: 10.1017/ CBO9780511542657.

JAMES, P.; MIDDLETON, S. The productivity and economic comparison of high-density production systems for 'Cripps Pink' and 'Cripps Red' apples in South Australia. Acta Horticulturae, v.903, p.611-618, 2011. DOI: 10.17660/ActaHortic.2011.903.84.

JUNG, S.-K.; CHOI, H.-S. Light penetration, growth, and fruit productivity in 'Fuji' apple trees trained to four growing systems. Scientia Horticulturae, v.125, p.672-678, 2010. DOI: 10.1016/j. scienta.2010.05.027.

KREUZ, C.L.; SOUZA, A.; PETRI, J.L. Impacto da intensificação da densidade de plantio na rentabilidade em duas cultivares de macieira em Fraiburgo-SC. Revista Brasileira de Fruticultura, v.28, p.240-243, 2006. DOI: 10.1590/S0100-29452006000200019.

LARSEN, F.E.; HIGGINS, S.S.; DOLPH, C.A. Rootstock influence over 25 years on yield, yield efficiency and tree growth of cultivars 'Delicious' and 'Golden Delicious' apple (Malus domestica Borkh.). Scientia Horticulturae, v.49, p.63-70, 1992. DOI: 10.1016/0304-4238(92)90143-Z.

LO BIANCO, R.; POLICARPO, M.; SCARIANO, L. Effects of rootstock vigour and in-row spacing on stem and root growth, conformation, and dry-matter distribution of young apple trees. Journal of Horticultural Science and Biotechnology, v.78, p.828-836, 2003. DOI: 10.1080/14620316.2003.11511705.

MUSACCHI, S.; ANCARINI, V.; GAMBERINI, A.; GADDONI, M.; GRANDI, M.; SANSAVINI, S. Response of training system, planting density and cultivar in pear. Acta Horticulturae, v.671, p.463-469, 2005. DOI: 10.17660/ActaHortic.2005.671.65.

PASA, M. da S.; FACHINELLO, J.C.; ROSA JÚNIOR, H.F. da; DE FRANCESCHI, E.; SCHMITZ, J.D.; SOUZA, A.L.K. de. Performance of 'Rocha' and 'Santa Maria' pears as affected by planting density. Pesquisa Agropecuária Brasileira, v.50, p.126-131, 2015. DOI: 10.1590/S0100-204X2015000200004.

PASA, M. da S.; FACHINELLO, J.C.; SCHMITZ, J.D.; SOUZA, A.L.K. de; FRANCESCHI, E. de. Desenvolvimento, produtividade e qualidade de peras sobre porta-enxertos de marmeleiro e Pyrus calleryana. Revista Brasileira de Fruticultura, v.34, p.873-880, 2012. DOI: 10.1590/S0100-29452012000300029.

PASA, M.S; EINHORN, T.C. Heading cuts and prohexadione-calcium affect the growth and development of 'd'Anjou' pear shoots in a high-density orchard. Scientia
Horticulturae, v.168, p.267-271, 2014. DOI: 10.1016/j. scienta.2014.02.003

PETRI, J.L.; LEITE, G.B.; COUTO, M.; FRANCESCATTO, P. Avanços na cultura da macieira no Brasil. Revista Brasileira de Fruticultura, v.33, p.48-56, 2011. Número especial.

POLICARPO, M.; TALLUTO, G.; LO BIANCO, R. Vegetative and productive responses of 'Conference' and 'Williams' pear trees planted at different in-row spacings. Scientia Horticulturae, v.109, p.322-331, 2006. DOI: 10.1016/j.scienta.2006.06.009.

PRAMANICK, K.K.; KISHORE, D.K.; SINGH, R.; KUMAR, J. Performance of apple (Malus $\mathrm{x}$ domestica Borkh.) cv. Red Spur on a new apple rootstock in high density planting. Scientia Horticulturae, v.133, p.37-39, 2012. DOI: 10.1016/j. scienta.2011.10.009.

R CORE TEAM. R: A language and environment for statistical computing., Vienna: R Foundation for Statistical Computing, 2014.

ROBINSON, T. Advances in apple culture worldwide. Revista Brasileira de Fruticultura, v.33, p.37-47, 2011a. Número especial.

ROBINSON, T.; HOYING, S.; SAZO, M.M.; DEMARREE, A.; DOMINGUEZ, L. A vision for apple orchard systems of the future. New York Fruit Quarterly, v.21, p.11-16, 2013.

ROBINSON, T.L. High density pear production with Pyrus communis rootstocks. Acta Horticulturae, v.909, p.259-269, 2011b. DOI: 10.17660/ActaHortic.2011.909.28.

ROM, R.C.; CARLSON, R.F. (Ed.). Rootstocks for Fruit Crops. New York: Wiley, 1987. 494p.

SANSAVINI, S.; ANCARANI, V.; NERI, D. Overview of intensive pear culture: planting density, rootstocks, orchard management, soil-water relations and fruit quality. Acta Horticulturae, v.800, p.35-50, 2008. DOI: 10.17660/ActaHortic.2008.800.1.

SANTOS, H.G. dos; JACOMINE, P.K.T.; ANJOS, L.H.C. dos; OLIVEIRA, V.A. de; LUMBRERAS, J.F.; COELHO, M.R.; ALMEIDA, J.A. de; CUNHA, T.J.F.; OLIVEIRA, J.B. de. (Org.). Sistema brasileiro de classificação de solos. 3.ed. rev. e ampl. Brasília: Embrapa, 2013. 353p.

TWORKOSKI, T.; MILLER, S. Rootstock effect on growth of apple scions with different growth habits. Scientia Horticulturae, v.111, p.335-343, 2007. DOI: 10.1016/j.scienta.2006.10.034.

VERHEIJ, E.W.M.; VERWER, F.L.J.A.W. Light studies in a spacing trial with apple on a dwarfing and a semi-dwarfing rootstock. Scientia Horticulturae, v.1, p.25-42, 1973. DOI: 10.1016/0304-4238(73)90004-6.

Recebido em 9 de julho de 2015 e aprovado em 28 de abril de 2016

Pesq. agropec. bras., Brasília, v.51, n.4, p.348-356, abr. 2016

DOI: $10.1590 / \mathrm{S} 0100-204 \mathrm{X} 2016000400007$ 